\title{
Coûts de transaction dans les modes de commercialisation de l'anacarde au nord-ouest du Bénin
}

\author{
Pamphile Kokou Degla ${ }^{1,2, *}$ et José Herbert Ahodode ${ }^{1}$ \\ ${ }^{1}$ Laboratoire d'Analyses et de Recherches sur les Dynamiques Economiques et Sociales (LARDES), 01 BP 123, Parakou, Bénin \\ ${ }^{2}$ Département d'Economie et de Sociologie Rurales, Faculté d'Agronomie, Université de Parakou, 01 BP 123, Parakou, Bénin
}

\begin{abstract}
Résumé - Du fait de son rôle dans l'obtention de revenus pour les paysans et de devises pour le pays, l'anacarde est une culture d'un fort intérêt économique et stratégique au Bénin. Sa commercialisation est cependant soumise à de nombreuses contraintes, dont les coûts de transaction qui font l'objet de la présente étude. En se basant sur un échantillon aléatoire de 122 producteurs choisis au nord-ouest du Bénin et en utilisant l'approche des coûts d'opportunité, l'efficacité transactionnelle des deux modes de commercialisation (individuelle et groupée) a été analysée. Il ressort des résultats que les producteurs supportent des coûts de transaction de $93 \mathrm{~F} \mathrm{CFA} / \mathrm{kg}$ dans le mode de vente individuelle contre seulement $43 \mathrm{~F} \mathrm{CFA} / \mathrm{kg}$ dans la vente groupée $(1 €=656 \mathrm{FCFA})$. Dans les deux modes, la composante majeure est constituée par les coûts de transaction ex ante $(98 \%$ ) contre seulement $2 \%$ pour les coûts expost. Si les résultats montrent que la vente groupée a un avantage comparatif en termes de réduction des coûts de transaction, ce mode peine cependant à se développer et le mode individuel reste toujours dominant (62\%). Ce résultat ne corrobore pas l'idée généralement admise de préférence des acteurs pour le mode de coordination minimisant les coûts de transaction dans un processus d'échange. Il suggère la nécessité d'approfondir l'analyse de l'efficacité transactionnelle, en incluant l'analyse de la perception des producteurs sur le fonctionnement de chaque mode de commercialisation et sur l'importance des coûts de transaction liés à chacun.
\end{abstract}

Mots clés : coûts de transaction / mode de coordination / noix de cajou / Bénin

Abstract - Transaction costs in the trading modes of cashew nuts in northwestern Benin. Because of its role in generating income for farmers and currencies for the country, cashew is a crop of economic and strategic interest in Benin. Its marketing, through two main channels (individual vs. grouped sales) is however subject to many constraints, including transaction costs which are the subject of this study. Based on a random sample of 122 producers chosen in northwestern Benin and using the opportunity cost approach, the transactional efficiency of the two contractual sales methods was analysed. The results show that $93 \mathrm{~F} \mathrm{CFA} / \mathrm{kg}$ of transaction costs are borne by the producers in the individual sales mode against only $43 \mathrm{~F} \mathrm{CFA} / \mathrm{kg}$ in the grouped sales mode $(1 \$=542 \mathrm{~F} \mathrm{CFA})$. The major components of transaction costs in both modes are the exante ones (98\%) while expost transaction costs represent only $2 \%$. Although the results show that group selling has the best comparative advantage in terms of reducing transaction costs, this mode is struggling to develop, and the individual mode is still dominant (62\%). Thus, this result does not corroborate the generally accepted idea that actors systematically choose the mode of coordination that minimizes transaction costs in an exchange process. However, it suggests the need to deepen the analysis of transactional efficiency in the future by including the analysis of producers' perception of the functioning of each marketing mode and the importance of the transaction costs incurred.

Keywords: transaction costs / mode of governance / cashew nuts / Benin

\section{Introduction}

Longtemps délaissés dans l'analyse économique, les coûts de transaction $(\mathrm{CT})$ connaissent un regain d'intérêt dû à

\footnotetext{
$\overline{\text { *Auteur de correspondance }}$ : pamphile.degla@yahoo.fr
}

l'émergence de l'économie néo-institutionnelle. En effet les postulats de base de la théorie économique néoclassique stipulent l'existence d'un marché parfait et pur où les individus sont supposés être rationnels dans leur comportement. Tous les échanges se font sans friction et leur coordination est régie par le mécanisme de prix. Avec l'hypothèse de concurrence 
parfaite et de rationalité économique des agents, le marché conduit naturellement vers un équilibre, lequel est atteint sans subir de coûts de fonctionnement (Chavance, 2007). Sur le marché règne une transparence de l'information et une homogénéité des biens échangés. Les contrats sont complets et ne laissent aucune marge de manœuvre à la fraude et la tricherie. Mais si cette fiction est nécessaire pour traduire en modèle les idées de base de l'efficience économique, les conditions d'un tel modèle se révèlent cependant de plus en plus éloignées du monde réel pour appréhender et expliquer, même approximativement, la complexité de la réalité économique (Göbel, 2002). Car dans la réalité, le fonctionnement du marché est imparfait du fait des incertitudes liées à l'hétérogénéité des biens échangés et de l'incertitude informationnelle. La coordination du prix basée sur l'hypothèse d'homogénéité et du marché parfait apparaît ainsi ne pouvoir prendre en compte toute la diversité des biens (Dieye, 2006). La plupart des marchés sont incomplets et fragmentés, l'information sur le prix se révèle souvent inexistante ou insuffisante, ou apparaît comme le sous-produit d'un autre type d'information (Ménard, 1995). Les contrats sont nécessairement incomplets à cause de l'asymétrie d'information et de l'hypothèse de la rationalité limitée des contractants, qui, de ce fait, ne peuvent anticiper toutes les contingences possibles. Ainsi ces imperfections du marché rendent son accès et son utilisation coûteux (Wang, 2003). Dans ces conditions, l'ensemble des coûts induits, appelés « coûts de transaction », sont les coûts de recherche et d'information, les coûts de négociation et de décision et les coûts de surveillance et d'exécution des contrats (Coase, 1937). Ces coûts, qui ne sont pas à confondre avec les coûts de production (Degla, 2012) et qui ne sont généralement pas pris en compte dans l'analyse économique néoclassique, sont pourtant des coûts réels dont l'existence influence le comportement des agents économiques et les amène, lors des échanges, à choisir l'arrangement institutionnel susceptible de réduire au mieux les CT (Williamson, 1991).

Au Bénin, dans le cas de la mise en marché de l'anacarde, la deuxième culture de rente après le coton et qui est également importante dans d'autres pays de la sous-région (Koffi et Oura, 2019; Ruf et al., 2019), la coordination des échanges se fait à travers deux principaux modes de commercialisation, la vente individuelle et la vente groupée. L'arrangement contractuel individuel revêt plusieurs aspects à cause de la multitude d'intermédiaires qui animent le circuit de vente, créant ainsi des arrangements contractuels emboîtés. L'asymétrie d'information relative au prix et aux conditions du marché est si forte qu'il n'est pas aisé pour les producteurs de faire exante le meilleur choix de partenaires contractants (sélection adverse). Profitant de leur avance informationnelle, les intermédiaires introduisent des contrats leur permettant de développer ex post des comportements opportunistes (aléa moral) et de tirer une rente comportementale. Face à ces situations, la vente groupée a été initiée pour faciliter la mise en marché des noix par les producteurs. Mais si la théorie des CT enseigne qu'à chaque mode de gouvernance correspond un niveau de CT donné, aucune étude n'a été jusqu'ici menée au Bénin pour déterminer effectivement la nature et le niveau des CT dans les deux principaux modes de mise en marché de l'anacarde. L'une des rares études existantes (Degla, 2012) porte certes sur l'analyse des CT dans la commercialisation des noix de cajou en général dans la zone d'étude, mais n'aborde pas spécifiquement le cas des différents arrangements institutionnels qui pourtant caractérisent le système complexe de vente de ces noix au Bénin. La présente étude se veut donc une application concrète, complémentaire, du concept des CT au Bénin.

\section{Matériel et méthodes}

\subsection{La théorie des coûts de transaction}

L'unité d'analyse dans l'approche des CT étant la transaction, la théorie des CT essaie d'expliquer quel arrangement institutionnel ou mode d'organisation a un avantage comparatif dans une transaction donnée (Slangen et al., 2008).

Selon cette théorie, les agents organisent leurs transactions au sein de structures de gouvernance qui visent à minimiser les CT résultant de la présence de risques contractuels (Royer, 2009). Ces risques proviennent de la juxtaposition d'hypothèses comportementales (rationalité limitée et opportunisme potentiel des agents économiques), et des attributs transactionnels (fréquence, incertitude et spécificité des actifs) (Williamson, 1991), mais aussi de l'environnement institutionnel, défini comme l'ensemble des règles formelles et informelles qui établissent la base pour la production, l'échange et la distribution (Davis et North, 1971). Outre les attributs transactionnels analysés par Williamson (1991), les travaux de Barzel (1982) mettent en évidence les problèmes de mesure des attributs en termes de coûts, comme par exemple celle de la qualité des biens échangés. Tous ces aspects étant liés à une transaction, ils déterminent alors l'ampleur et la nature des CT et ce faisant aussi les avantages comparatifs du mécanisme de transaction (Williamson, 1991).

\subsection{Approche de la mesure des coûts de transaction}

Dans la littérature économique, le concept des CT est sujet à diverses interprétations qui n'ont pas favorisé l'émergence d'une approche unique de mesure. Parmi les différentes approches existantes, l'approche des coûts d'opportunité de Benham et Benham (2005) associée à l'approche des «Nonmarket transaction costs » de North (1987) ont été retenues à cause de leur pertinence pour la présente étude, dont l'objectif impose une estimation directe des CT.

\subsection{Estimation des coûts de transaction dans les deux modes de vente}

L'estimation des CT s'est faite pour les deux modes courants de transaction, la vente individuelle et la vente groupée. Pour chacun, les CT ont été classés en deux types : exante et expost. Les coûts exante regroupent les coûts générés lors des phases de collecte d'information, de négociation et de signature du contrat; les coûts expost se rapportent à ceux générés lors de l'exécution du contrat (Tab. 1). Ainsi les CT estimés ne concernent que les coûts directement liés au processus de commercialisation, ce qui exclut les coûts indirects tels que ceux liés au développement de relations personnelles, de réseau, d'habiletés spécifiques à la transaction et, dans le cas de la coopérative, les coûts initiaux 
Tableau 1. Typologie des coûts de transaction.

Table 1. Typology of transaction costs.

\begin{tabular}{|c|c|c|}
\hline & $\begin{array}{l}\text { Coûts de transaction } \\
\text { ex ante }\end{array}$ & $\begin{array}{l}\text { Coût de transaction } \\
\text { ex post }\end{array}$ \\
\hline Vente individuelle & $\begin{array}{l}\text { CO du temps de: } \\
\text { - recherche de l'acheteur } \\
\text { - négociation, signature de contrat } \\
\text { - transport des noix du verger au domicile } \\
\text { ou magasin de l'acheteur } \\
\text { Frais éventuels de: } \\
\text { - communication avec l'acheteur } \\
\text { - autres frais non numéraires liées à la vente des noix }\end{array}$ & $\begin{array}{l}\text { Cas paiement par tranche: } \\
\text { - frais de communication pour réclamation } \\
\text { - CO du temps de déplacement pour récupérer } \\
\text { l'argent } \\
\text { - coût éventuel dans la gestion de conflits en } \\
\text { cas de non paiement }\end{array}$ \\
\hline Vente groupée & $\begin{array}{l}\text { CO du temps consacré par la coopérative pour la: } \\
\text { - préparation des activités de précampagne de noix } \\
\text { — mobilisation de fonds auprès de banques pour préfinancer } \\
\text { l'achat des noix aux producteurs } \\
\text { - temps de collecte de noix au magasin } \\
\text { - recherche, négociation et signature de contrat avec l'acheteur } \\
\text { - organisation des réunions et autres } \\
\text { - communication avec les membres } \\
\text { CO du temps individuel de: } \\
\text { - participation aux réunions, formation relative aux } \\
\text { conditions d'achat et de dépôt des noix } \\
\text { - convoyage des noix du champ au magasin } \\
\text { Frais individuels de: } \\
\text { - communication, repas et autres dans le cadre des } \\
\text { réunions liées à la vente } \\
\text { - transport du membre du domicile vers le lieu de } \\
\text { réunion/formation }\end{array}$ & $\begin{array}{l}\text { CO du temps consacré par la coopérative au: } \\
\text { - suivi et à l'exécution du contrat de vente } \\
\text { - règlement éventuels conflits } \\
\text { Coût de communication individuelle: } \\
\text { - après dépôt des noix au magasin pour le suivi } \\
\text { de la vente } \\
\text { - pour récupérer l'argent } \\
\text { - coût éventuel de gestion de litiges }\end{array}$ \\
\hline
\end{tabular}

$\mathrm{CO}=$ Coût d'opportunité.

Source: Résultats d'enquête, 2018.

de création et de mise en œuvre de cette structure. Ces derniers coûts peuvent d'ailleurs, comme le suggère Royer (2009), être considérés comme amortis après un certain nombre d'années. Au niveau de chaque producteur individuel, le temps consacré en minutes ou en heures et les dépenses monétaires (communication, déplacements vers les clients, etc.) effectuées à chaque étape du processus ont été enregistrés. Quant aux membres de la coopérative, au temps collectif de préparation de la campagne, de mobilisation de fonds, de recherche, de négociation et de signature de contrat et autres démarches relatives à la vente des noix, recensé et réparti équitablement entre les membres, s'ajoutent le temps individuel de participation aux différentes réunions et les dépenses monétaires (communication, déplacement vers les centres de réunions, achat de nourriture lors des réunions, etc.) effectuées dans le cadre des réunions et autres activités liées à la vente des noix (Tab. 1).

Dans la comparaison des deux modes, la vente individuelle a été considérée comme un mode homogène malgré ses trois variantes (vente au champ/à domicile/au marché). En effet, la vente à domicile représentant plus de $95 \%$ de l'ensemble des ventes individuelles, les deux autres variantes ont été assimilées au cas dominant pour faciliter la comparaison avec la vente groupée qui ne s'effectue qu'au niveau du magasin de la coopérative. Les avantages et inconvénients de chacun des deux modes ont été résumés dans le tableau 2, dont les informations proviennent aussi bien de la revue de littérature que des entretiens en «focus groups» et des observations personnelles faites sur le terrain.

En s'appuyant sur Degla (2012), dont le modèle fonctionnel utilise aussi bien le coût d'opportunité que les dépenses monétaires, les CT générés à chaque phase du processus de vente ont été exprimés sous la forme de l'équation (1). Étant donné que dans toute exploitation agricole, la main-d'œuvre de l'exploitant ne peut être allouée qu'à deux principales utilisations alternatives (dans l'exploitation ou hors de celle-ci pour un travail salarié), le coût d'opportunité de la main de l'exploitant est alors considéré comme étant le taux de rémunération de la main-d'œuvre (Kay et al., 2008). Nous avons donc retenu le salaire agricole moyen dans la zone d'étude. Ainsi on a: 
Tableau 2. Forces et faiblesses des deux modes de vente.

Table 2. Strengths and weaknesses of the two sales methods.

\begin{tabular}{|c|c|c|}
\hline Modes de vente & Forces & Faiblesses \\
\hline
\end{tabular}

* = Voir Göbel (2002).

Source: Résultats d'enquête 2018 auprès des producteurs, des coopératives et autres personnes ressources.

$$
C T 1_{J}=\sum_{I=1}^{P} w T_{I J}+C_{I J}
$$

Avec CT1 le CT exante, $w$ le salaire agricole dans la zone d'étude, $T_{I J}$ le temps consacré par le producteur $i$ pour une opération donnée dans le mode de vente $j, P$ le nombre total de producteurs, égal à l'unité dans le cas de la vente individuelle et variant de 1 à $\mathrm{N}$ dans le cas de la vente groupée, et $C_{I J}$ les charges monétaires (communications et autres dépenses) engagées par le producteur i dans le mode de vente $j$.

Par analogie le CT expost (CT2) peut s'exprimer comme suit :

$$
C T 2_{J}=\sum_{I=1}^{P} w T_{I J}+C_{I J} .
$$

Ainsi le CT total pour chaque mode de vente est:

$$
C T_{J}=C T 1_{J}+C T 2_{J} .
$$

Les CT ont été estimés en $\mathrm{kg}$ de noix et comparés grâce au test $T$ de Student.

\subsection{Zone d'étude, échantillonnage et base de données}

L'étude s'est déroulée dans la commune de Kouandé, l'une des zones de forte production d'anacarde du pays. Située au nord-ouest du Bénin dans le département de l'Atacora (Fig. 1), cette commune s'étend sur $4500 \mathrm{~km}^{2}$ de savane aux conditions pédoclimatiques très favorables à l'anacardier. Dix villages ont été choisis pour leur importance dans la production d'anacarde; 122 producteurs pratiquant soit la vente individuelle (77) soit la vente groupée (45) y ont été choisis au hasard à partir d'une liste fournie par le service agricole. Les données primaires collectées sont de nature quantitative et qualitative, et sont relatives aux caractéristiques socioéconomiques des producteurs, au temps consacré à chaque étape du processus de vente, aux dépenses monétaires engagées lors de l'organisation de la vente des noix, aux quantités de noix vendues, aux prix de vente, aux rapports avec les différents acteurs, aux types de contrat, etc. Elles ont été collectées par le biais d'une enquête semi-structurée basée sur un questionnaire individuel et des entretiens de groupe. Des observations participantes ont été aussi utilisées. Les données secondaires portant sur des informations générales relatives à la production et à la commercialisation des noix dans la zone ont été collectées auprès des centres de documentation et de personnes ressources. Les données ont été analysées en utilisant le logiciel Stata 12.0.

\section{Résultats}

\subsection{Caractéristiques socio-économiques des producteurs}

Les interviewés ont un âge moyen de 47 ans $( \pm 11)$, la plupart sont de sexe masculin $(85 \%)$ et mariés $(88 \%)$. 


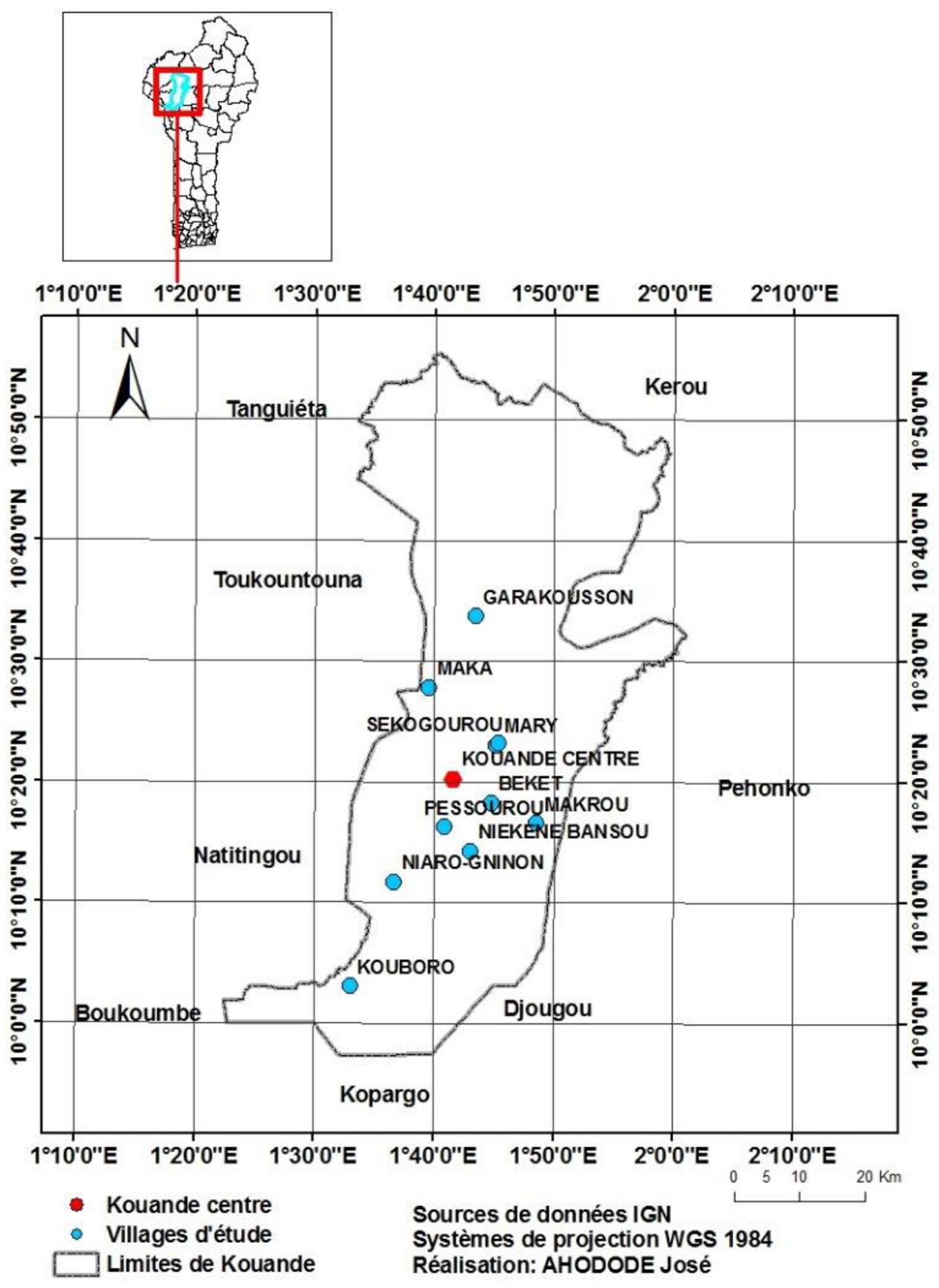

Fig. 1. Zone d'étude.

Fig. 1. Study area.

La majorité ( $52 \%)$ ne sont affiliés à aucune association et n'ont aucun accès au crédit formel $(71 \%)$. L'instruction ne concerne que $18 \%$ des interviewés, $10 \%$ ayant un niveau dépassant le primaire. Le nombre moyen d'actifs par producteur est de 6 , ce qui constitue un potentiel de main-d'œuvre familiale suffisant pour assurer les travaux dans des plantations dont la taille moyenne est de 2,3 ha $( \pm 4,2)$, puisque la plupart des interviewés $(81 \%)$ n'utilisent que rarement la main-d'œuvre salariée dans leurs anacarderaies. En dehors de la taille des exploitations, chacune des autres variables quantitatives utilisées varie très peu d'un groupe de producteurs à un autre (Tab. 3).

\subsection{Modes de coordination de la vente d'anacarde}

Contrairement au coton, suivi et contrôlé de la production jusqu'à la commercialisation par des structures étatiques ou para-étatiques, le marché de l'anacarde est peu organisé et chaque année l'intervention de l'État se résume à la fixation du prix plancher et à l'annonce du début et de la fin de la période de commercialisation. Le marché de l'anacarde concerne une multitude de petits producteurs face à un nombre plus faible d'acheteurs. Parmi les acheteurs, on retrouve les exportateurs étrangers, les grossistes, les semi-grossistes et de nombreux intermédiaires nationaux complexifiant le circuit 
Tableau 3. Caractéristiques socio-économiques des producteurs.

Table 3. Socioeconomic characteristics of producers.

\begin{tabular}{|c|c|c|c|c|c|c|c|}
\hline \multicolumn{2}{|c|}{ Caractéristiques des producteurs } & \multicolumn{4}{|c|}{ Type de vente pratiquée } & \multicolumn{2}{|l|}{ Ensemble } \\
\hline & & Ind & Grp & Ind & Grp & $\frac{\text { Fréquence }}{\text { Ind }+ \text { Grp }}$ & $\frac{\%}{\text { Ind }+ \text { Grp }}$ \\
\hline \multirow{2}{*}{ Sexe } & Hommes & 70 & 34 & 91 & 76 & 104 & 85 \\
\hline & Oui & 15 & 7 & 19 & 16 & 22 & 18 \\
\hline \multirow[t]{3}{*}{ Niveau instruction } & Non & 62 & 38 & 81 & 84 & 100 & 82 \\
\hline & Total & 77 & 45 & 100 & 100 & 122 & 100 \\
\hline & Célibataire & 13 & 2 & 17 & 4 & 15 & 12 \\
\hline Situation matrimoniale & Marié & 64 & 43 & 83 & 96 & 107 & 88 \\
\hline \multirow{2}{*}{ Accès crédit } & Total & 77 & 45 & 100 & 100 & 122 & 100 \\
\hline & Comptant & 69 & 39 & 90 & 87 & 14 & 12 \\
\hline \multirow[t]{3}{*}{ Mode paiement } & Différé & 8 & 6 & 10 & 13 & 108 & 88 \\
\hline & Total & 77 & 45 & 100 & 100 & 122 & 100 \\
\hline & Oui & 23 & 22 & 30 & 51 & 45 & 37 \\
\hline \multirow[t]{4}{*}{ Association culturale } & Non & 54 & 23 & 70 & 49 & 77 & 63 \\
\hline & Total & 77 & 45 & 100 & 100 & 122 & 100 \\
\hline & Groupe des Producteurs & $\mathbf{N}$ & Moyenne & Min & & Maximum & Écart type \\
\hline & Ind & 77 & 47 & 26 & & 75 & 11,4 \\
\hline Âge producteur & Grp & 45 & 48 & 26 & & 73 & 9,3 \\
\hline Actifs ménage & Ind & 77 & 4 & 1 & & 9 & 1,4 \\
\hline \multirow[t]{3}{*}{ Main-d'œuvre salariée } & Grp & 45 & 4 & 0 & & 9 & 1,7 \\
\hline & Ensemble & 122 & 4 & 0 & & 9 & 1,5 \\
\hline & Ind & 77 & 1,6 & 0,3 & & 6 & 1,1 \\
\hline \multirow[t]{2}{*}{ Taille exploitation (ha) } & Grp & 45 & 3,5 & 0,5 & & 40 & 6,6 \\
\hline & Ensemble & 122 & 2,3 & 0,3 & & 40 & 4,2 \\
\hline
\end{tabular}

Ind = Producteurs pratiquant la vente individuelle ; Grp = Producteurs pratiquant la vente groupée.

Source : Résultats d'enquête, 2018.

de commercialisation de plus de $95 \%$ de la quantité totale d'anacarde produite chaque année dans la zone d'étude et estimée à environ $9500 \mathrm{t}$, soit $13 \%$ de la production nationale.

Le mode de coordination dominant est la vente individuelle, pratiqué par $62 \%$ des producteurs interrogés. Dans cet arrangement contractuel individuel, l'acheteur et le producteur se recherchent et aboutissent à un contrat d'achat. Mais si pour la négociation, les informations de base d'accès au marché telles que celles relatives au «prix plancher», aux critères d'appréciation de la qualité des noix et aux autres conditions du marché, devraient être disponibles pour toutes les parties prenantes, nos résultats montrent une maîtrise très inégale de ces informations basiques. En effet, la plupart des informations d'accès au marché sont d'origine étatique et ne sont facilement accessibles que pour les acheteurs, du fait de leur niveau d'instruction et de leur facilité d'accès aux structures administratives. La plupart des producteurs sont illettrés $(82 \%)$ et éparpillés dans des hameaux; ils ignorent aussi bien 
l'existence de ces informations de base que leur source. Ce qui limite l'alternative de choix des meilleurs clients pour ces petits producteurs. Vu leur avance informationnelle, les acheteurs arrivent sur le terrain en position de force avec un pouvoir de négociation leur permettant d'imposer les prix d'achat et de spéculer sur la qualité des noix qu'ils définissent et manipulent à leur guise, comme l'affirment $62 \%$ des vendeurs individuels interrogés qui déclarent que la décision finale relative à la qualité des noix et au prix ne revient le plus souvent qu'aux acheteurs. Même lors des pesées, leur meilleure connaissance des appareils de mesure offre aux acheteurs une marge de manœuvre pour abuser de l'ignorance de petits producteurs analphabètes. Soucieux de garantir leur approvisionnement, certains acheteurs pratiquent des contrats de long-terme incluant le préfinancement (sous forme de crédit remboursable) de l'entretien des plantations et même des besoins monétaires des producteurs, comme c'est le cas pour environ $35 \%$ des vendeurs individuels interrogés. Malheureusement, ces pratiques de fidélisation créent, du fait des crédits alloués, des situations de dettes permanentes, empêchant certains petits producteurs de profiter d'éventuelles améliorations ultérieures de prix sur le marché, étant donné que les acheteurs n'offrent que de bas prix pour mieux s'assurer des différents risques (perte de qualité, fluctuation de prix, mévente des noix etc.) liés à leur activité. Dans le même temps, le respect strict de ces contrats par certains producteurs n'est pas toujours assuré, car nombre d'entre eux trouvent aussi les moyens de contourner les contrats en cédant informellement une partie de leur stock à des clients jugés momentanément plus offrants. Pour $5 \%$ des petits vendeurs individuels interrogés, c'est au premier acheteur qu'ils bradent leur produit pour régler les problèmes financiers immédiats auxquels ils sont confrontés.

Face à toutes ces difficultés, des initiatives visant à organiser collectivement la vente se sont développées depuis quelques années. Cela s'est traduit par la création de coopératives de services dans lesquelles les producteurs adhérents apportent leurs noix. Les coopératives négocient alors directement avec les exportateurs sur la base de conditions de prix et de qualité connues de tous et parviennent à un accord au profit des membres de la coopérative. Malgré les avantages qu'offre ce mode de coordination en termes de garantie de prix de vente, de nonbradage des stocks de noix, de vente unique des stocks, de réduction de l'asymétrie informationnelle, etc., force est de constater qu'il peine à se développer, à cause de l'ignorance de nombreux petits producteurs de l'existence même d'un tel mode alternatif de vente et de ses avantages, mais aussi de la méfiance de certains producteurs vis-à-vis de la question coopérative, suite à des cas de malversations financières vécues dans le passé avec d'anciens groupements villageois. Même si de nos jours les risques de détournement sont limités dans ces nouvelles coopératives du fait de la mise en place de divers organes de contrôle, la hantise des mauvaises expériences du passé continue de freiner l'élan de bon nombre de ces petits producteurs pour y adhérer malgré les avantages qu'elles offrent.

\subsection{Niveaux de production des noix et chiffres d'affaires}

Outre les coûts de transaction (CT), l'analyse des résultats montre que $1,81 \mathrm{t}$ de noix de cajou ont été commercialisées par producteur en vente groupée, contre seulement $0,71 \mathrm{t}$ en vente individuelle. Si en moyenne le prix accordé aux pratiquants de la vente groupée est de $500 \mathrm{FCFA} / \mathrm{kg}$, il n'est que de $492 \mathrm{~F} \mathrm{CFA} / \mathrm{kg}$ chez les producteurs utilisant la vente individuelle, avec un minimum de $342 \mathrm{FCFA} / \mathrm{kg}$ pour les 2 catégories et un maximum de $583 \mathrm{~F} \mathrm{CFA} / \mathrm{kg}$ pour les ventes individuelles et de $600 \mathrm{FCFA} / \mathrm{kg}$ pour les adhérents des coopératives au cours des trois dernières années. Au total, la vente des noix a rapporté un chiffre d'affaires annuel moyen de 982099 FCFA aux adhérents de la vente groupée contre $356301 \mathrm{~F} \mathrm{CFA}$ aux vendeurs individuels (Tab. 4).

\subsection{Niveaux des coûts de transaction dans les différents modes de vente}

La vente de l'anacarde a généré en moyenne pour chaque producteur $75 \mathrm{~F} \mathrm{CFA} / \mathrm{kg}$ de CT. Ces coûts sont de $93 \mathrm{~F} \mathrm{CFA} / \mathrm{kg}$ pour la vente individuelle et de $43 \mathrm{FCFA} / \mathrm{kg}$ pour la vente groupée. En considérant chaque mode et chacune des composantes des $\mathrm{CT}$, les résultats montrent que $91 \mathrm{~F} \mathrm{FCA} / \mathrm{kg}$ ont été générés exante par la vente individuelle contre $42 \mathrm{~F} \mathrm{CFA} / \mathrm{kg}$ par la vente groupée. De même, $2 \mathrm{FCFA} / \mathrm{kg}$ de CT expost ont été engendrés par la vente individuelle contre moins de $1 \mathrm{~F} \mathrm{CFA} / \mathrm{kg}$ par la vente groupée. Les CTengendrés par la vente individuelle dépassent donc, à tous les niveaux, ceux générés par la vente groupée (Tab.4). De l'examen des différents éléments constitutifs des CT, il ressort que, au niveau des vendeurs individuels, l'élément générant le plus de CT dans la composante ex ante est la recherche d'acheteurs et la négociation, avec $61 \%$ de ces CT exante, suivie du temps de regroupement des noix ( $28 \%$ ) et du temps d'acheminement éventuel des noix vers le magasin du client $(10 \%)$. Au niveau des membres de la coopérative, c'est le temps de réunion qui est le poste le plus élevé (45\%) du total des CT exante, suivi du temps de recherche et de négociation avec les clients (25\%), du temps de formalités après le dépôt des noix $(16 \%)$ et de celui du convoyage des noix au magasin de la coopérative (12\%) (Tab. 5). Quant à la composante expost, c'est la gestion de conflits qui est le poste générant le plus de CTex post $(95 \%)$ pour les vendeurs individuels, suivi du temps de récupération de l'argent après-vente $(4 \%)$. Pour les membres des coopératives, ce sont les frais de communication après-vente qui constituent la principale source de CT expost (59\%), suivis du temps de formalité après-vente (41\%).

\section{Discussion}

De la taille des anacarderaies et du volume de noix de cajou vendu par producteur, il ressort que les membres des coopératives sont en moyenne, dans la zone d'étude, de plus grands producteurs que les vendeurs individuels. Le prix d'achat offert à ces producteurs en vente groupée différant de celui offert aux vendeurs individuels, les adeptes de la vente groupée jouissent d'un avantage comparatif dans la réalisation de leur chiffre d'affaires comme le montrent les résultats ci-dessous présentés. Dans le contexte actuel de revenu agricole saisonnier incertain chez la plupart des producteurs, du fait des effets de plus en plus manifestes du changement climatique dans la zone d'étude (Agbossou et al., 2012), et compte tenu de l'importance du revenu issu de la production 
Tableau 4. Coûts de transaction pour chaque mode de vente.

Table 4. Transaction cost according to the sales mode.

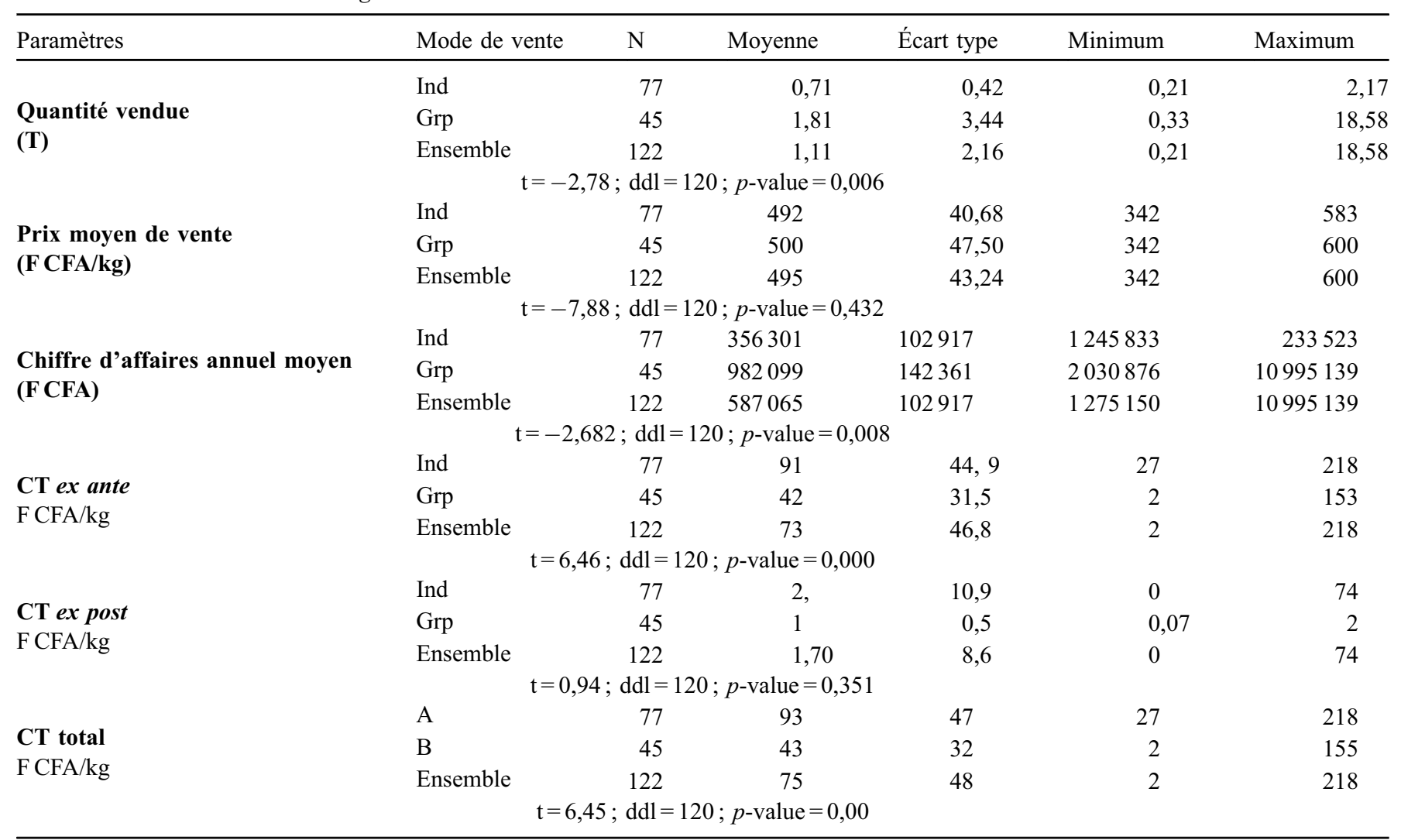

Ind = Vente individuelle; Grp = Vente groupée.

Source : Résultats d'enquête, 2018.

Tableau 5. Proportions détaillées des coûts de transaction issus des éléments constitutifs des composantes ex ante et ex post.

Table 5. Part of transaction costs resulting from ex ante and ex post components in detail.

\begin{tabular}{|c|c|c|c|c|}
\hline \multirow{2}{*}{ Eléments constitutifs des composantes de $\mathrm{CT}$} & \multicolumn{2}{|c|}{ Vendeurs individuels } & \multicolumn{2}{|c|}{ Membres coopérative } \\
\hline & $\begin{array}{l}\text { ex ante } \\
(\%)\end{array}$ & $\begin{array}{l}\text { ex post } \\
(\%)\end{array}$ & $\begin{array}{l}\text { ex ante } \\
(\%)\end{array}$ & $\begin{array}{l}\text { ex post } \\
(\%)\end{array}$ \\
\hline Temps de recherche et de négociation & 61 & & 25 & \\
\hline Temps regroupement des noix & 28 & & & \\
\hline Gestion Conflits & & 95 & & \\
\hline Autres & 1 & 1 & & \\
\hline Temps de réunion & & & 45 & \\
\hline Frais communication & & & 1 & \\
\hline Déplacement vers lieu réunion & & & 1 & \\
\hline Total & 100 & 100 & 100 & 100 \\
\hline
\end{tabular}

$\mathrm{CT}=$ Coûts de transaction.

Source : Résultats d'enquête, 2018. 
d'anacarde pour les ménages ruraux (Koffi et Oura, 2019; Ruf et al., 2019), cette différence significative dans le chiffre d'affaires issu de la vente des noix entre les adeptes de la vente groupée et les vendeurs individuels contribue à rendre la situation financière des adhérents de la coopérative meilleure que celle des vendeurs individuels.

En ce qui concerne les CT, les résultats montrent que, dans l'ensemble, c'est le mode de vente individuelle qui a généré le plus de CT ( $93 \mathrm{~F} \mathrm{CFA} / \mathrm{kg}$ ), plus du double de ceux de la vente groupée $(43 \mathrm{~F} \mathrm{CFA} / \mathrm{kg})$. Il ressort également de ces résultats que la composante essentielle des CT générés lors de la mise en marché de l'anacarde provient des coûts exante (98\%). Au sein de ces CT ex ante, ceux du mode de vente individuelle sont plus élevés $(91 \mathrm{FCFA} / \mathrm{kg})$ que ceux de la vente groupée ( $42 \mathrm{~F} \mathrm{CFA} / \mathrm{kg})$. La différence est significative $(p=0,00)$, et est due surtout au coût d'opportunité du temps individuel de recherche et de négociation avec les acheteurs, qui est collectif dans la vente groupée et ne coûte donc que peu par individu. Contrairement aux membres des coopératives qui pratiquent une vente unique de tout leur stock de noix, la plupart des vendeurs individuels procèdent le plus souvent à des ventes multiples, ne vendant à chaque fois qu'une partie de leur stock en espérant trouver les jours suivants des clients plus offrants. Dès qu'ils sont au courant de la présence d'un acheteur supposé être plus offrant dans le village, ils cèdent encore une partie de leur stock. Et le processus continue jusqu'à ce que tout leur stock de noix s'épuise. Même chez les producteurs liés par des contrats, c'est-à-dire des relations personnalisées avec des acheteurs donnés, il n'est pas rare que ces producteurs recherchent d'autres clients pour vendre une partie de leurs noix, faisant ainsi du temps de recherche de partenaires et de négociation une source importante de CT exante. Pour les membres des coopératives, c'est le temps de réunion relative à l'organisation de la vente des noix qui constitue le poste générant le plus de CT ex ante. Outre le temps d'acheminement des noix qu'on retrouve au niveau des vendeurs individuels en termes de «temps d'acheminement des noix vers les magasins des clients» et au niveau des membres des coopératives en termes de «temps d'acheminement des noix vers les magasins de la coopérative » comme un autre élément de CT exante, on observe au niveau des membres des coopératives un poste particulier, le «temps de formalités après dépôt », constituant une source non négligeable de CT exante et qui pourrait agir comme un frein à l'adhésion aux coopératives pour des producteurs ayant généralement déjà une aversion très prononcée pour les formalités administratives.

Le fait que le mode de vente individuelle génère plus de CT (93 F CFA $/ \mathrm{kg}$ ) pour les producteurs que le mode de vente groupée ( $43 \mathrm{~F} \mathrm{CFA} / \mathrm{kg}$ ), met en évidence, tout comme Royer (2009) dans ses travaux relatifs à la mise en marché du lait au Québec, non seulement l'importance du mode de coordination collective dans la vente de produits agricoles, mais aussi l'existence de nombreux problèmes qui minent la commercialisation des noix dans la zone d'étude. En effet, contrairement au marché du coton, celui de l'anacarde est peu réglementé et sujet à des dysfonctionnements. Ainsi, l'absence de réseaux adéquats de diffusion aux producteurs d'informations de base (annonce du début de la saison, prix plancher, etc.) conduit à une asymétrie informationnelle ne profitant qu'à la plupart des acheteurs, instruits et plus habitués aux structures administratives et au jeu du marché. Pendant que ces derniers accèdent facilement à l'information sur les conditions du marché, les petits producteurs ignorent l'existence même de ces informations et leurs sources; la recherche d'information est très difficile pour ces petits producteurs exposés ainsi à des risques tels que la sélection adverse, le hold-up sur le prix et la qualité des noix que les acheteurs sont le plus souvent les seuls à juger et à imposer. Ces types de problèmes, largement décrits dans la théorie de l'Agence ou la théorie des coûts de l'information (Göbel, 2002), deviennent de plus en plus manifestes dans la commercialisation des noix dans la zone d'étude et conduisent à des situations affectant les différents acteurs. Ainsi, si l'asymétrie informationnelle amène certains acheteurs à abuser de la précarité financière d'un certain nombre de producteurs, en les entraînant de fait dans des dettes permanentes par le biais de contrats de campagne répétitifs basés sur des octrois de crédits en tous genres et dont le but est de fidéliser les producteurs (cas de $35 \%$ des vendeurs individuels), la défaillance du système offre aussi une marge de manœuvre à certains producteurs pour développer des comportements opportunistes (non-respect des clauses de contrats préétablis, ventes parallèles des noix, etc.), au détriment de leurs partenaires contractuels.

$\mathrm{Si}$, dans l'ensemble, ces résultats confirment l'existence réelle des problèmes théoriques décrits dans la littérature des CT et dans la théorie de l'Agence (Göbel, 2002) avec l'exposition des principaux acteurs aux différents risques, ils mettent aussi en évidence l'importance relative d'un mode de gouvernance donné (ici le mode de commercialisation), mais ne corroborent pas l'hypothèse d'alignement émise par Williamson (1985) selon laquelle les acteurs économiques vont chercher à choisir le mode de gouvernance qui, pour un ensemble d'attributs transactionnels, minimisent les CT. Si dans la présente étude, le mode de vente groupée s'avère être le mode de gouvernance réduisant au mieux les CT et offrant même les meilleurs avantages économiques comparatifs, il peine à se développer depuis plus d'une décennie et le mode de vente individuelle reste toujours dominant. La différence observée ici par rapport à l'hypothèse fondamentale de Williamson, qui toutefois garde sa validité dans l'analyse économique de l'organisation de la production et des processus d'échange, ne pourrait être expliquée que par l'existence d'un certain nombre de facteurs spécifiques au mode de vente groupée dans la zone d'étude, susceptibles de modifier dans une certaine mesure les effets attendus dans la théorie. Il s'agit notamment des contraintes techniques, organisationnelles et financières liées à ce mode de commercialisation. Tout d'abord la vente groupée relève de l'initiative de certaines ONG et autres acteurs de développement en milieu rural qui tentent de promouvoir ce mode de vente. Mais partant du fait que la large adoption d'une innovation en milieu rural peut fortement dépendre de l'importance accordée à la co-construction de cette innovation avec les bénéficiaires (Latzko-Toth, 2010), il est possible que la non-implication d'un grand nombre de producteurs dans la conception et la mise en œuvre de cette nouvelle approche de vente de noix puisse justifier l'ignorance ou l'indifférence de nombreux producteurs par rapport à ce mode de vente groupée. Les lourdeurs dans la gestion administrative et financière des coopératives à travers les 
nombreuses formalités en amont et en aval du processus de mise en marché des noix pourraient aussi être un frein à l'adhésion d'un certain nombre de producteurs, plus soucieux de leur liberté de décision et d'action. Plus dissuasifs encore pourraient être aussi les contraintes financières en termes de frais d'adhésion et de parts sociales de valeur non négligeable dont tout adhérent aux coopératives doit s'acquitter. Si le mauvais souvenir de certaines malversations financières vécues dans le passé dans de nombreuses formes coopératives reste intact chez certains producteurs, suscitant ainsi leur méfiance vis-à-vis de tout autre mouvement coopératif, ce sont plutôt les relations de parenté entre certains producteurs et acheteurs qui dictent à d'autres producteurs leur désintérêt pour l'actuelle action coopérative. L'importance de toutes ces contraintes impose que des actions susceptibles de les lever soient entreprises par les décideurs impliqués dans la promotion de la vente groupée. Des actions en termes d'information et de sensibilisation des vendeurs individuels sur les avantages qu'offre la vente groupée mais aussi en termes de réduction des lourdeurs dans le fonctionnement des coopératives contribueraient sûrement à améliorer considérablement l'adoption de ce mode de vente dans la zone d'étude. D'un autre côté, les résultats actuels suggèrent aussi la nécessité d'une étude plus approfondie de l'efficacité transactionnelle des différents modes, susceptible de prendre en compte non seulement des facteurs non abordés dans la présente étude (la nature et l'ampleur des CT), mais aussi une analyse de la perception des acteurs, et notamment des producteurs, du fonctionnement des différents modes de vente de noix et de l'importance des CT qu'ils subissent. Il serait également nécessaire d'étudier la part que prend l'opportunité d'accéder à un crédit dans la décision de vente individuelle sous contrat.

\section{Conclusion}

L'analyse de l'efficacité transactionnelle des deux modes, individuel et collectif, de vente de l'anacarde au nord-ouest du Bénin montre que la vente individuelle génère $93 \mathrm{FCFA} / \mathrm{kg}$ de CT contre seulement $43 \mathrm{FCFA} / \mathrm{kg}$ pour la vente groupée. Comptant pour $98 \%$ de ces montants, les CT exante sont la composante essentielle des CT dans chacun des modes. Malgré le fait que la vente groupée minimise les CT générés dans la mise en marché des noix, elle reste cependant le mode de vente le moins répandu depuis plusieurs années, ce qui corrobore très peu l'idée généralement admise de préférence des acteurs pour le mode le plus réducteur de CT dans un processus d'échange. Ce résultat montre donc qu'il n'est pas suffisant d'analyser l'efficacité transactionnelle d'un mode de coordination sous le seul angle de la réduction des CT. Il est aussi nécessaire de prendre en compte un certain nombre de facteurs spécifiques à la zone d'étude. Dans la perspective de futures recherches complémentaires, une analyse plus approfondie de l'efficacité transactionnelle globale incluant la nature et l'ampleur des CT au niveau de tous les acteurs impliqués dans chacun des modes, ainsi que la perception par les producteurs du fonctionnement de chaque mode et de l'importance des
CT générés s'impose. Il conviendrait aussi d'analyser le rôle des avantages liés à un mode spécifique, comme la possibilité de l'accès au crédit dans le cadre de contrats individuels.

\section{Références}

Agbossou EK, Toukon C, Akponikpè PBI, Afouda A. 2012. Climate variability and implications for maize production in Benin: A stochastic rainfall analysis. African Crop Science Journal 20(s2): 493-503.

Barzel Y. 1982. Measurement cost and the organization of market. Journal of Law and Economics 25(1): 27-48. DOI: 10.1086/467005.

Benham A, Benham L. 2005. Measuring the costs of exchange. In: Ménard C, ed. Institutions, contracts and organisations: Perspectives from New Institutional Economics. Cheltenham (UK): Edward Elgar, pp. 367-375.

Chavance B. 2007. L'économie institutionnelle. Paris (France) : Ed. La Découverte, Collection Repères. 128 p. DOI: 10.3917/dec. chava.2007.01.

Coase RH. 1937. The nature of the firm. Economica 4: 386-405. DOI: 10.3917/dec.chava.2007.01.

Davis LE, North CDD. 1971. Institutional change and American economic growth. Cambridge (UK): Cambridge University Press. DOI: $10.1017 / \mathrm{CBO} 9780511561078$.

Degla KP. 2012. Transaction costs in the trading system of cashew nuts in the North of Benin: A field study. American Journal of Economics and Sociology 71: 277-297. DOI: 10.1111/ j.1536-7150.2012.00830.x.

Dieye PN. 2006. Arrangements contractuels et performance des marchés du lait local au sud du Sénégal. Thèse de doctorat, École Nationale Supérieure Agronomique de Montpellier, France, 211 p. https://afrique-ouest.cirad.fr/content/download/6764/62883/ver sion/1/file/obj_5248_file_obj_7351_file_These_PNDIEYE_2006. pdf [2019/08/09].

Göbel E. 2002. Neue Institutionen-ökonomik, Konzeption und betriebswirtschaftliche Anwendungen. Stuttgart: Lucius \& Lucius, 400 p.

Kay RD, Edwards WM, Duffy P. 2008. Farm management, 6th ed. McGraw-Hill-International Editions, 480 p.

Koffi SY, Oura KR. 2019. Les facteurs de l'adoption de l'anacarde dans le bassin cotonnier de Côte d'Ivoire. Cahiers Agricultures 28 (24): 1-8. DOI: 10.1051/cagri/2019025.

Latzko-Toth G. 2010. La co-construction d'un dispositif sociotechnique de communication: le cas de l'Internet Relay Chat. Sociologie. Thèse de doctorat en communication, Université du Québec à Montréal, $421 \mathrm{p}$.

Ménard C. 1995. L'économie des organisations. Paris (France) : La Découverte, Collections Repères, $126 \mathrm{p}$.

North DC. 1987. Institutions, transaction costs and economic growth. Economy Inquiry 25(3): 419-428. DOI: 10.1111/j.1465-7295.1987. tb00750.x.

Royer A. 2009. Analyse économique néo-institutionnelle de la mise en marché collective. Application au secteur laitier du Québec. Economies et Finances. Université Panthéon-Sorbonne-Paris I, $350 \mathrm{p}$. https://tel.archives-ouvertes.fr/tel00402464 [2019/06/06].

Ruf F, Kone S. Bebo B. 2019. Le boom de l'anacarde en Côte d'Ivoire: transition écologique et sociale des systèmes à base de coton et de cacao. Cahiers Agricultures 28(21): 1-12. DOI: $10.1051 /$ cagri/2019019. 
Slangen LHG, Loucks LA, Slangen AHL. 2008. Institutional economics and economic organisation theory: An integrated approach. Wageningen (The Netherlands): Wageningen Academic Publishers, $431 \mathrm{p}$. Wang N. 2003. Measuring transaction costs: An incomplete survey. The Ronald Coase Institute. www.coase.org/w-wang2003measu ringtransactioncosts.pdf [2019/09/19].
Williamson OE. 1985. The economic institutions of capitalism. NewYork (USA): The Free Press.

Williamson OE. 1991. Comparative economic organization. The analysis of discrete structural alternatives. Administrative Science Quaterly 36: 269-296. DOI: 10.1086/467284.

Citation de l'article : Degla PK, Ahodode JH. 2021. Coûts de transaction dans les modes de commercialisation de l'anacarde au nord-ouest du Bénin. Cah. Agric. 30: 22. 